\title{
Testicular microlithiasis and dyspermia: Is there any correlation?
}

\author{
Francesco Catanzariti, Ubaldo Cantoro, Vito Lacetera, Giovanni Muzzonigro, Massimo Polito \\ Polytechnic University of Marche, Faculty of Medicine, Department of Odontostomatologic and Specialized Clinical Sciences, \\ Urology Clinic, Department of General and Specialized Surgery, University Hospital, Ancona, Italy.
}

\begin{abstract}
Summary Background: Testicular microlithiasis (MT) is an uncommon sonographic finding (prevalence in the literature: 0.7 to 6\%). Several studies have highlighted its possible correlation with an increased risk of testicular cancer, but few studies have investigated its possible link with dyspermia. Objectives: The aim of our study was to investigate in our series the number of patients with microlithiasis, diagnosed by ultrasound, and compare the quality of their sperm with that of patients in a control group with normal testicular ultrasound exam.

Materials and methods: We performed 277 consecutive testicular ultrasound examinations from January 2012 to July 2012. Among all these, we selected 86 patients that showed no pathological elements at echography and 11 patients affected by MT, to one or both testicles. Each patient was also submitted to a short-term semen analysis using the WHO2010 parameters for sperm evaluation. Results: Among 11 patients with MT, 7 (63.63\%) were dyspermic and 4 (36.36\%) were normospermic. Among the 86 patients with normal testicular ultrasound 51 (59.3\%) were dyspermic, 4 (4.65\%) were azoospermic, while the remaining 31 (36.05\%) were normospermic. Comparing the results of the two groups we obtained an odds ratio of 0.99 (95\% CI: 0.27 to 3.64, p: 0.98).

Conclusions: This study, although preliminary, with a low number of partecipants, shows that sperm quality is not affected by the presence of testicular microlithiasis, because the results of spermiograms are almost comparable between the two groups.
\end{abstract}

KEY WORDS: Testicular microlithiasis; Infertility; Testicular ultrasound.

Submitted 1 September 2013; Accepted 31 December 2013

\section{INTRODUCTION}

Testicular microlithiasis (MT) is a quite rare ultrasound evidence, with a low prevalence, from 0.7 to $6 \%$, as described in literature (1-3), although with the evolution of ultrasound machines, which allow the identification of structures of diameter less than one millimeter, the prevalence of MT has been increasing.

The MT is characterized by the presence of hyperechoic spots of diameter between 1 and $3 \mathrm{~mm}$, which don't have posterior shadow cone, within testicular parenchima.

Microlithiasis can be classified as "classic" or "limited" depending on the presence of more or less than 5 hyperechoic spots per ultrasound section (Figure 1).

Several studies in literature have shown a correlation between MT and some pathological conditions, such as testicular cancer, cryptorchidism, varicocele, testicular torsion, epididymitis, orchitis, Klinefelter's syndrome, male pseudohermaphroditism, neurofibromatosis and HIV infection (4).

Some papers studied the association between microlithiasis and testicular cancer (4) and showed that the MT can predispose to the development of a carcinoma in situ (CIS) or a germ cell tumors (TGCT) of the testis.

Other authors have also noted that there could be a correlation between MT and intratubular germ cell neoplasia of unclassified type (ITGCNU) (5) and that testicular microlithiasis might be part of a complex disease, the testicular dysgenesis syndrome (TDS), comprising in addition to the testicular microlithiasis also other features such as infertility, testicular atrophy, cryptorchidism, TGCT and other abnormalities of sexual development (6).

In literature there are other studies, less numerous and with conflicting results, which analyze the association between infertility and testicular microlithiasis.

Some studies support the theory that there would be a higher prevalence of testicular microlithiasis among infertile patients (7), compared to fertile men. This evidence should be more frequent in the classical forms than in limited MT and the pathogenetic mechanism underlying this correlation has been described by some Authors as the result of reduced inflow of arterial blood caused by microlithiasis (8).

Other authors instead believe that not statistically significant correlation exists between MT and infertility (9) and exclude that there is a greater number of antisperm antibodies in patients with testicular microlithiasis (10), which could be the cause of infertility in men affected by MT. 
Figure 1.

A. Classic microlithiasis.

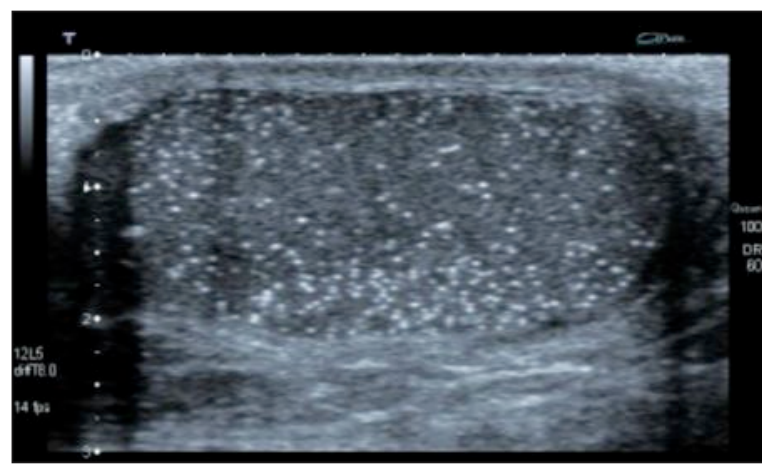

B. Limited microlithiasis.

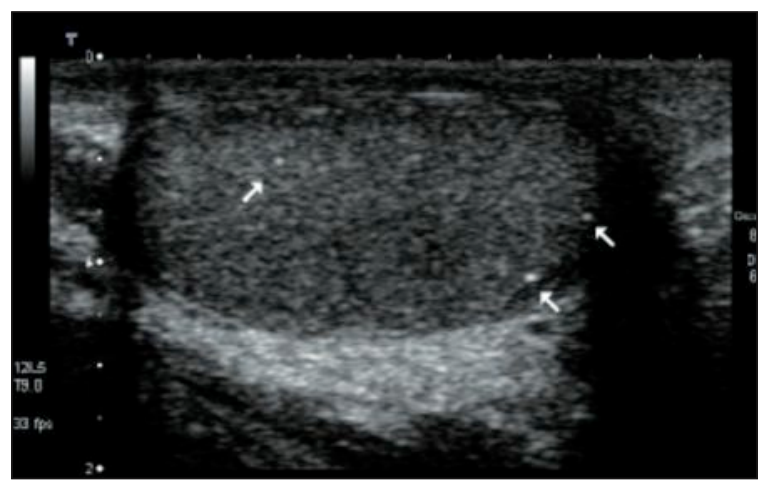

\section{Objectives}

The aim of our work was to study the incidence of microlithiasis in our series, but above all to analyze how this ultrasound evidence can affect sperm quality of patients with this testicular desease, compared to patients normal at testicular ultrasound.

\section{Materials AND METHOdS}

From January 2012 to July 2012, we performed 277 consecutive testicular ultrasound at our Urology Clinic. The testicular ultrasound examinations were performed by three different operators, all with good experience in the field of ultrasound, with the same new generation of machine, using a $7.5 \mathrm{MHz}$ linear probe.

During the examination particular attention has been placed on detection of hyperechoic areas compatible with testicular MT, distinguishing the limited forms (less than 5 hyperechoic areas for ultrasound section of diameter between 1 and $3 \mathrm{~mm}$ without shadow rear) from the classical ones (at least 5 hyperechoic areas for ultrasound section of diameter between 1 and $3 \mathrm{~mm}$ without shadow cone rear).

Among all the examinations, we selected those patients with testicular ultrasound without no alterations of didymus and epididymis (control group) and those with the presence of classical MT at ultrasound (study group). Patients with limited MT were eliminated from the study. Each patient of both groups was then subjected to a semen analysis that was performed in a short time (within 30 days) from the ultrasonographic examination.
The semen analysis was conducted after at least 3 days of sexual abstinence. The semen samples were collected in the hospital asking patients to deposit its seed directly in sterile $120 \mathrm{ml}$ containers. The samples were analyzed within 1 hour from ejaculation.

After liquefaction, semen volume was measured by a syringe with an accuracy of $0.1 \mathrm{ml}$.

The sperm concentration and motility was then evaluated by optical microscope through Makler chamber with a magnification of x 200. We used WHO 2010 parameters for the sperm evaluation.

\section{RESULTS}

Among the 277 testicular ultrasound examinations, we selected 11 (3.97\%) patients with classical MT and 86 (31.05\%) patients with normal testis at ultrasound investigation (Figure 2), while we eliminated from the study the remaining 180 patients who resulted affected by other testicular diseases (varicocele, cysts, inflammation, etc.) at echography.

\section{Figure 2.}

Results in 277 consecutive testicular ultrasounds.

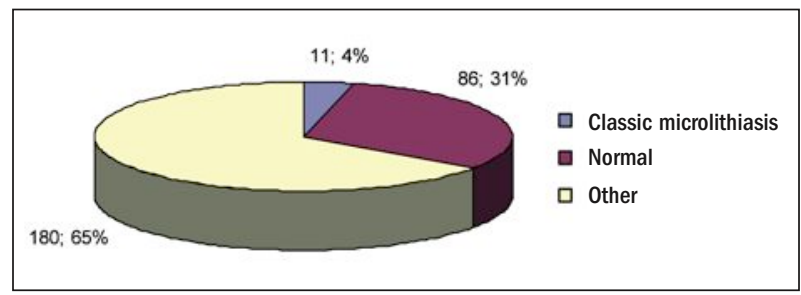

Spermiograms among patients with classical MT showed 7 cases (63.63\%) of dyspermia, defined as the presence of at least one of the three parameters (concentration, motility and forms) under reference values according to the WHO 2010 classification. The remaining 4 patients (36.36\%) of the study group instead resulted with normal semen analysis.

Among the patients belonging to the control group we found dyspermia in 51 (59.3\%), azoospermia in 4 (4.65\%) and normospermia in 31 (36.05\%) cases (Figure 3).

\section{Figure 3.}

Results of spermiograms in patients affected by MT and normal at echography.

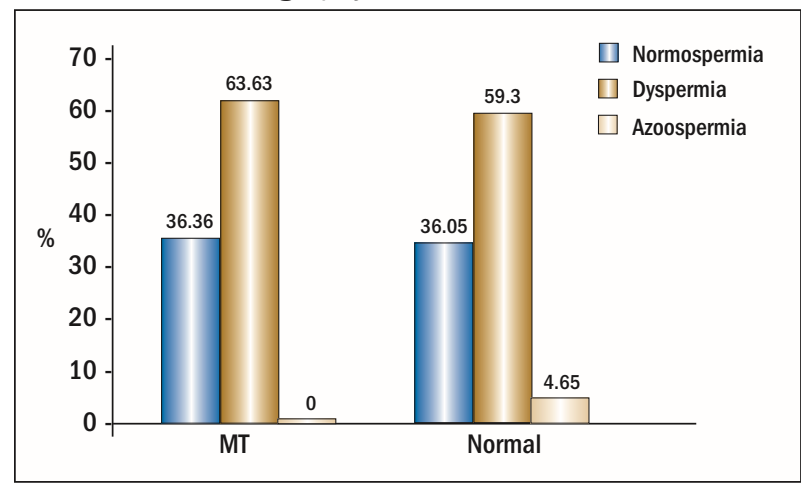


By comparing the results of the two groups and calculating the odds ratio, we discovered a value of 0.99 (95\% CI: 0.27 to 3.64 , P: 0.98 ), so that the percentage distribution of dyspermia in the two groups resulted substantially comparable between the two groups and thus the testicular microlithiasis does not seem to determine the presence of dyspermia.

\section{Conclusions}

Our study showed that the testicular microlithiasis is an ultrasound fairly rare evidence, indeed, the prevalence in our series has remained around $4 \%$, similar to that described in the literature.

Moreover, we have shown that the testicular microlithiasis not lead to changes in sperm quality, therefore results of spermiograms of patients with normal testes at ultrasound study were similar to those of the MT group.

However, our study has several limitations: testicular ultrasound exams as well as semen analysis were performed by different operators so that results may have been affected by the interoperator variability. Another important limitation is the low number of patients in the study group, justified by the fact that MT is an unusual disease so that it is difficult to perform a study with a high number of patients.

Further evaluation with a larger study population and greater standardization for both ultrasound of the testis and sperm evaluation would be necessary to demonstrate our thesis and to reach statistically significant conclusions about the correlation between MT and dyspermia, although our work is one of the few in the literature who analyzed this connection.

\section{References}

1. Yee WS, Kim YS, Kim SJ, et al. Testicular microlithiasis: prevalence and clinical significance in a population referred for scrotal ultrasonography. Korean J Urol. 2011; 52:172-7.

2. Dutra RA, Perez-Bóscollo AC, Melo EC, Cruvinel JC Clinical importance and prevalence of testicular microlithiasis in pediatric patients. Acta Cir Bras. 2011; 26:387-90.

3. Peterson AC, Bauman JM, Light DE, et al. The prevalence of testicular microlithiasis in an asymptomatic population of men 18 to 35 years old. J Urol 2001; 166:2061-2064.

4. Van Casteren NJ, Looijenga LH, Dohle GR. Testicular microlitiasis and carcinoma in situoverview and proposed clinical guideline. Int J Androl. 2009; 32:279-287.

5. Tan IB, Ang KK, Ching BC, et al. Testicular microlithiasis predicts concurrent testicular germ cell tumors and intratubular germ cell neoplasia of unclassified type in adults: a meta-analysis and systematic review. Cancer. 2010; 116:4520-32.

6. Tan MH, Eng C. Testicular microlithiasis: recent advances in understanding and management. Medscape. Nat Rev Urol. 2011; 8:153-63.

7. Wang PY, Shen MY. Testicular microlithiasis: ultrasonic diagnosis and correlation with male infertility. Zhonghua Nan Ke Xue. 2009; 15:158-60.

8. Deng CH, Liu GH, Lü JY, et al. Testicular microlithiasis influences seminal profile and testicular blood flow in infertile men. Zhonghua Nan Ke Xue. 2008; 14:606-9.

9. Yee WS, Kim YS, Kim SJ, et al. Testicular microlithiasis: prevalence and clinical significance in a population referred for scrotal ultrasonography. Korean J Urol. 2011; 52:172-7.

10. Jiang $H$, Zhu WJ. Testicular microlithiasis is not a risk factor for the production of antisperm antibody in infertile males. Andrologia. 2013; 45:305-9.

\section{Correspondence}

Francesco Catanzariti, MD (Corresponding Author)

fracatanzariti@libero.it

Resident in Urology

Ubaldo Cantoro, MD

ubaldocantoro@tiscali.it

Resident in Urology

Vito Lacetera, MD

vlacetera@gmail.com

Urologist

Giovanni Muzzonigro, MD

g.muzzonigro@univpm.it

Professor of Urology, Chief Department of Urology

Massimo Polito, MD

max_polito@virgilio.it

Urologist, Chief Department of Uro-Andrology

Polytechnic University of Marche, Faculty of Medicine,

Department of Odontostomatologic and Specialized Clinical Sciences,

Urology Clinic, Department of General and Specialized Surgery,

University Hospital, Via Conca 71 - I-60020 Ancona, Italy 